\title{
OFDM Channel Estimation Algorithm Research and Simulation in Tunnel Environment
}

\author{
Ting Wang ${ }^{1}$, Ming Lv ${ }^{2}$ \\ ${ }^{1}$ Department of Electronic Engineering, University of Electronic Science and Technology of China, Sichuan, China \\ ${ }^{2}$ Department of Electronic Engineering, University of Electronic Science and Technology of China, Sichuan, China \\ wangting_4002@foxmail.com, lvming@uestc.edu.cn
}

\begin{abstract}
Least square (LS) algorithm based on least square criterion has poor estimation performance under low signal to noise ratio, if we directly use this method in tunnel environment, it will lead to larger estimation error. Considering the defects of the LS algorithm, this paper propose an improved LS channel estimation algorithm based on DFT time domain filtering. Firstly, this method will remove noises outside the guard interval; then, by setting a dynamic threshold, noises within the guard interval will be removed. Simulation results show that this method can achieve better estimation performance in low signal-to-noise ratio by selecting appropriate threshold, and this method is suitable for channel estimation in tunnel.

Index Terms - Tunnel, Channel estimation, Least square criterion, Discrete Fourier Transform (DFT), Threshold.
\end{abstract}

\section{Introduction}

The application of orthogonal frequency division multiplexing(OFDM) technology in tunnel environments has attracted wide attention in recent years, for its good performance under conditions of multi-path interference and fading. Channel estimation technique is one of the key technologies of OFDM systems, so in order to use OFDM technology in practical application, it is necessary to solve the problem of channel estimation.

LS channel estimation algorithm based on least square criterion, and the minimum mean square error(MMSE) channel estimation algorithm which promises minimum mean square error are the common algorithms of channel estimation [1]. Reference [2] and [3] analyzed and compared the two algorithms above respectively, and by analyzing the simulation results the author has found that: MMSE algorithm not only has high computation complexity, but also assumed the second-order statistics of channel is known, and such assumption is unpractical in the reality; the LS channel estimation algorithm has low computation complexity, and can be implemented without any prior information, so the LS channel estimation algorithm is more commonly used in practice. Tunnel environment is very complex, the wireless signal will be subjected to various disturbances when propagate in a tunnel channel, and thus leading to low signalto-noise ratio(SNR) of received signal [4]. The harsher conditions of tunnel channel make the LS estimation algorithm which proves to be useful previously inapplicable. In order to solve the problem of channel estimation under tunnel environment, this paper proposes an improved LS channel estimation method based on DFT time domain filtering, and simulated the method by MATLAB simulation platform. Simulation results show that, in the condition of low signal-tonoise ratio the performance of the proposed method has been greatly improved compared with LS algorithm, and is suitable for tunnel channel estimation.

\section{OFDM System Model}

Fig. 1 is the equivalent baseband diagram of OFDM system. As can be seen from fig.1, the high-speed serial binary information sequences will become relatively low rate parallel signals, after the digital baseband modulation and serial-toparallel conversion processing. Pilot symbols are inserted into the parallel signals and after an IFFT algorithm is performed also, the signal can be expressed as:

$$
\{x(n)\}=\operatorname{IFFT}\{x(k)\}=\sum_{k=0}^{N-1} X(k) \exp (j 2 \pi k / N), n=0,1, \cdots, N-1
$$

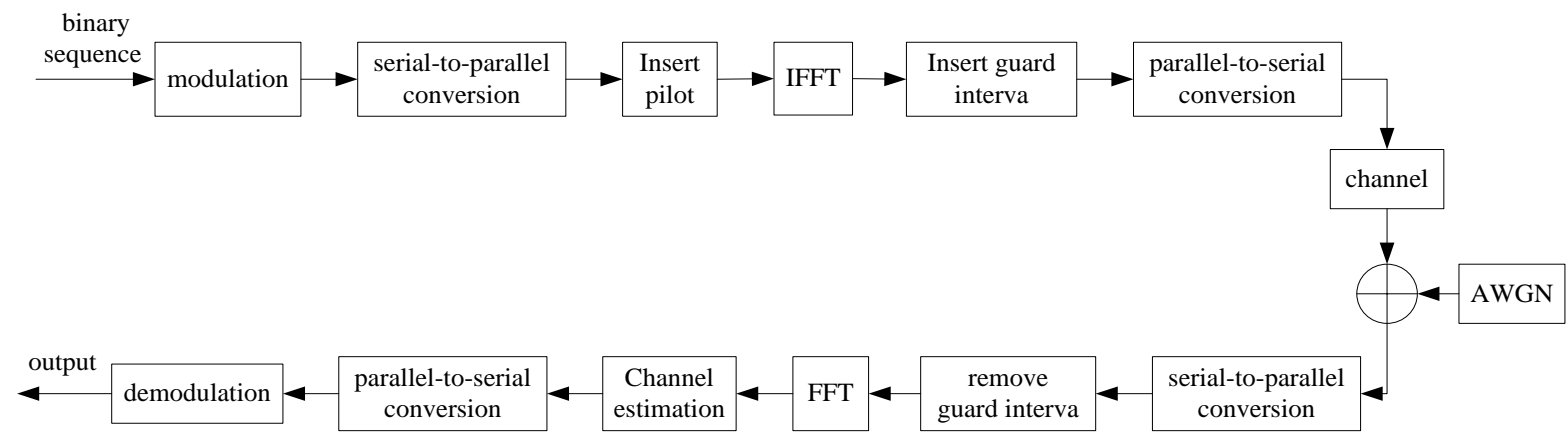

Fig. 1 The equivalent baseband diagram of OFDM system 
In the above formula, $N$ is the total number of sub-carriers; $X(k)$ is the transmitted signal on the $k^{\text {th }}$ subcarrier. Then, a guard interval is inserted into the transmitted signals and after transforming the parallel signals into a serial one, the processed signals will be emitted. On the other hand, the receiver will first convert the serial signals into a parallel one, then remove the guard interval and perform FFT algorithm, and after all the above steps, signal $Y(k)$ can be represented by the following formula [5].

$$
Y(k)=X(k) H(k)+W(k), k=0,1, \cdots, N-1
$$

Where $H(k)$ is the frequency response of the $k^{\text {th }}$ sub-carrier channel; $W(k)$ is the additive white Gaussian noise superimposed on $k^{\text {th }}$ sub-carrier.

\section{III.Channel Estimation Method Based on DFT Time Domain Filtering}

In the LS channel estimation algorithm, for which the pilot sequence is required, the estimated frequency response of the channel in the place of pilot symbol is assumed to be $\hat{H}_{P}$, then the expression of $\hat{H}_{P}$ can be written as follows:

$$
\hat{H}_{P}=X_{P}^{-1} Y_{P}=H_{P}+W_{P} / X_{P}
$$

Where $X_{P}$ and $Y_{P}$ are transmitted and received pilot signal in frequent domain, $H_{P}$ is true frequency response of the channel in the place of pilot symbol. $W_{P}$ is the noise super imposed on the pilot symbol. Equation (3) show that the estimation error of LS algorithm is mainly affected by noises. When the value of signal-to-noise ratio is low or the noise is large, the estimation deviation is greater. Thus, denoising as an extra step should be added in LS algorithm, if better estimation results are required.

Considering the defects of the LS algorithm, estimation method based on DFT time domain filtering is proposed to work under low signal-to-noise ratio environments. Because DFT can be realized with FFT technology in application, the new algorithm proposed has good practicability [6]. The main idea of the algorithm is: in OFDM systems, the length of the finite impulse response of time domain channel should be less than the length of guard interval, and the signal energy is mainly focused on the minority sampling points of protection interval [7]. Based on the previous analysis, the signal outside the protection interval can be viewed as noises and replaced by zero, thus complete the process of denoising. The diagram of the traditional LS channel estimation algorithm based on DFT time domain filtering is shown in fig. 2 .

As shown in fig. 2, the exact process of LS channel estimation algorithm based on DFT time domain filtering is: firstly, IFFT algorithm is applied to the results of LS estimation, and the transformed signal is:

$$
\hat{h}_{p}(n)=\operatorname{IFFT}\left[\hat{H}_{p}(k)\right]=h_{p}(n)+w(n), n=0,1, \cdots, N-1
$$

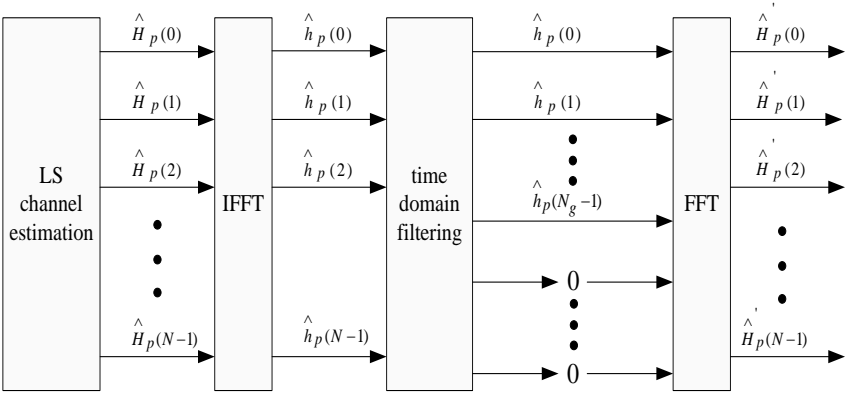

Fig. 2 The diagram of channel estimation based on DFT time domain filtering

Where $h_{p}(n)$ is the true impulse response of channel, $w(n)$ is the estimation error introduced by different kinds of noises. Commonly, the time domain impulse response is assumed to be finite, and signal power is focused on protection interval, signals outside protection interval can be regard as caused by noises. Thus equation (4) can be rewritten as:

$$
\hat{h}_{p}(n)=\left\{\begin{array}{cc}
h_{p}(n)+w(n), 0 \leq n \leq N_{g}-1 \\
w(n) & , N_{g} \leq n \leq N-1
\end{array}\right.
$$

Where $N_{g}$ is the length of protection interval. Then signals outside protection interval can be replaced by zeros by using a low pass filter. The filtered signal is:

$$
\hat{h}_{p}(n)=\left\{\begin{array}{cc}
h_{p}(n)+w(n), & 0 \leq n \leq N_{g}-1 \\
0 & , N_{g} \leq n \leq N-1
\end{array}\right.
$$

At last, FFT algorithm is applied to the filtered results, and the value of the LS channel estimation based on DFT time domain filtering is obtained. The final signal can be expressed as:

$$
\hat{H}_{p}^{\prime}(k)=\sum_{n=0}^{N-1} \hat{h}_{p}(n) \exp (-j 2 \pi n k / N), k=0,1, \cdots N-1
$$

After the above filtering process, the average power of noises reduced to $N_{g} / N$ times of original power. However, it can be seen from (6) that the algorithm can only handle noises outside protection interval, and noises inside protection interval remains. For further denoising inside protection interval, this paper proposes an improved algorithm for the traditional LS channel estimation algorithm based on DFT time domain filtering.

\section{Improved Channel Estimation Method Based on DFT Time Domain Filtering}

It can be learned from (6) that after time-domain filtering, noises outside the protection interval are wiped out, and the signal within protection interval is composed by two parts, one is the true value of the channel impulse response and the other part is the noise. Therefore, after time-domain filtering, the estimation error is mainly caused by noises within the 
protection guard. In order to further reduce estimation error and improve accuracy, it is necessary to further reduce the influence of the noise within the guard interval. Aimed to solve this problem, an improved channel estimation method based on DFT time-domain filtering is proposed.

The improved method reduces the noise within the protection interval by setting a threshold filter, i.e., after filter out the noise outside of the protection interval, we will further reduce the noise within the protection interval by selecting an appropriate threshold, thus achieve better estimation performance. The basic idea of this method is explained as follows: in a finite impulse response channel, the signal energy is mainly focused on the minority sampling points. For the emitted signals with certain power, when the system is in the low signal-to-noise ratio, noise power is larger; when the system is in the high signal-to-noise ratio, the noise power is smaller. Based on these ideas, we can choose a dynamic threshold to deal with noises within the protection interval. For example, the signal that is greater than the dynamic threshold will be reserved, and the signal less than the dynamic threshold will be treated as noise and reset to zero [8]. The block diagram of the improved method based on time-domain filter is shown in fig. 3 .

The detailed steps of the improved method are described as follows: first, the LS estimation value will be disposed by IFFT algorithm, and the transformed signal can be expressed as

$$
\hat{h}_{p}(n)=\frac{1}{N} \sum_{k=o}^{N-1} \hat{H}_{P}(k) \exp (j 2 \pi n k / N), n=0,1, \cdots, N-1
$$

Then the transformed signal will go through a time domain filter. Which is achieved by setting the value outside the guard interval to zero and selecting an appropriate threshold value to denoising the signal within the guard interval, the processed signal can be expressed as

$$
\hat{h}_{p}^{\prime}(n)=\left\{\begin{array}{c}
\hat{h}_{p}(n),\left|\hat{h}_{p}(n)\right|^{2}>\Gamma \\
0, \quad \text { others }
\end{array}\right.
$$

Where, $\Gamma=\alpha \bullet \beta, \alpha$ is the dynamic modulation factor, $0<\alpha<0.5$, and the value of $\beta$ is obtained by (10)

$$
\beta=\frac{1}{N_{g}} \sum_{n=0}^{N_{g}-1}\left|\hat{h}_{p}(n)\right|^{2}
$$

As can be seen from the above equation, dynamically change the value of the threshold can effectively suppress noises within the guard interval under different signal-to-noise ratio conditions.

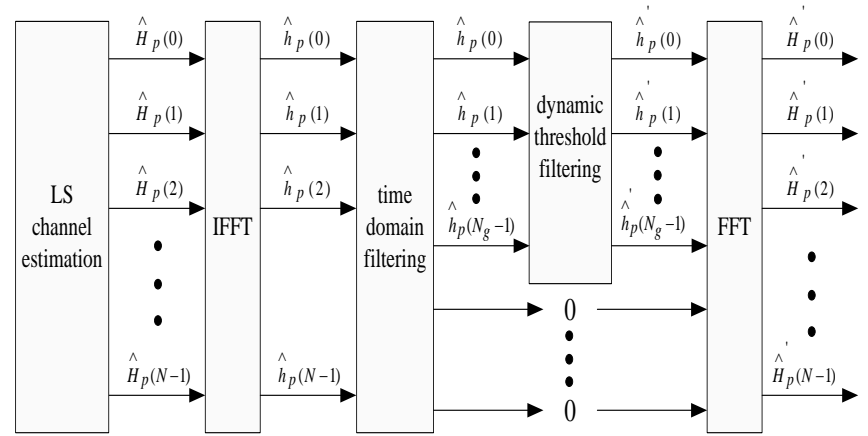

Fig. 3 The diagram of improved channel estimation based on DFT time domain filtering

At last, perform FFT to the filtering results, and the estimation value can be expressed as

$$
\hat{H}_{p}(k)=\sum_{n=0}^{N-1} \hat{h}_{p}^{\prime}(n) \exp (-j 2 \pi n k / N), k=0,1, \cdots N-1
$$

All the above procedure composed the new algorithm, and the frequency response of channel will thus be obtained finally.

\section{V . Simulation and Results Analysis}

In this paper, the Monte-Carlo method has been adopted to verify the performances of different channel estimation algorithms in tunnel environment. Parameters setting in the simulation are as follows: the subcarrier number is 128,100 OFDM symbols, the length of protection interval is 16 , the 16QAM modulation and block pilot with pilot interval 5 is employed.

The channel model adopted is Nakagami model, which is said to be applicable to tunnels of wireless signal propagation. For the model, Nakagami parameter $\mathrm{m}=2$, the frequency of carrier is $1 \mathrm{GHZ}$, the travelling speed of receiver is set to be $120(\mathrm{~km} / \mathrm{h})$, and the number of multipath is 16 .

Fig. 4 and fig. 5 give the mean square error(MSE) curve when $\alpha$ is 0.15 and 0.35 respectively. The curve named DFT plots the simulation results when denoising only carried outside protection interval, and the curve named improved DFT plots the simulation results the denoising carried both outside and inside protection interval. Analysis the figures, when $\mathrm{SNR}=2$ the DFT time domain estimation algorithm got $7 \mathrm{~dB}$ improvement compared with LS estimation algorithm, further improvement is obtained when dynamical threshold is used for noise reduction within protection interval. In addition, fig. 3 and fig. 4 also have shown that dynamically changing the value of $\alpha$ can effectively filter noises in different signal-tonoise ratio. For example, when the value of signal-to-noise ratio is low, large $\alpha$ would be preferable, and when the noise is comparatively small, a small $\alpha$ is supposed to be better. Thus in real application, $\alpha$ can be changed according to different signal-to-noise ratio environment, and superior estimation performance would be reached. 


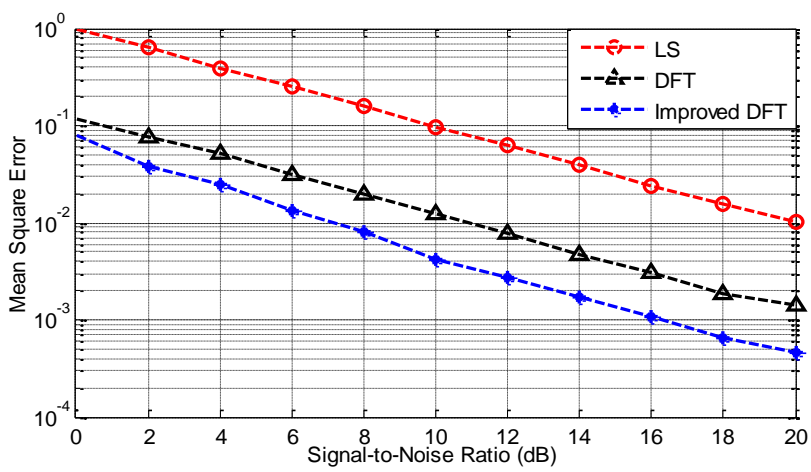

Fig. 4 Mean square error comparison with $\alpha=0.15$

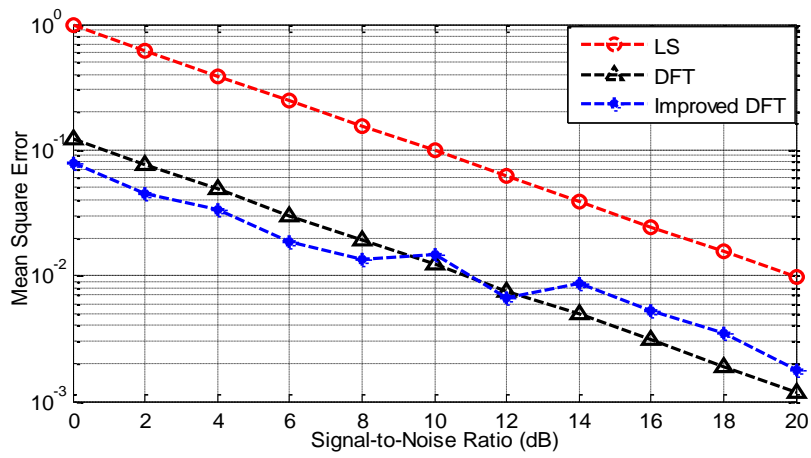

Fig. 5 Mean square error comparison with $\alpha=0.35$

Fig. 6 and fig. 7 are the frequency response and magnitude error of different estimation methods respectively with $\alpha=0.15, \mathrm{SNR}=5 \mathrm{~dB}$. Comparing both frequency response and magnitude error, it's obvious that estimation based on improved DFT algorithm got minimum deviation with real value, and which is 4 times lower than LS estimation algorithm. Thus we conclude that, the improved algorithm we proposed has superior estimation performance compared with existed methods, and can be applied to estimate channel in tunnel environment.

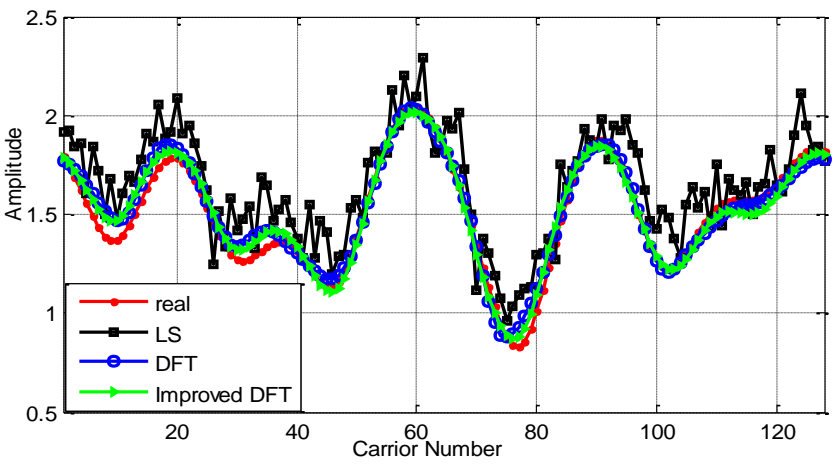

Fig. 6 Frequency response estimation of different methods

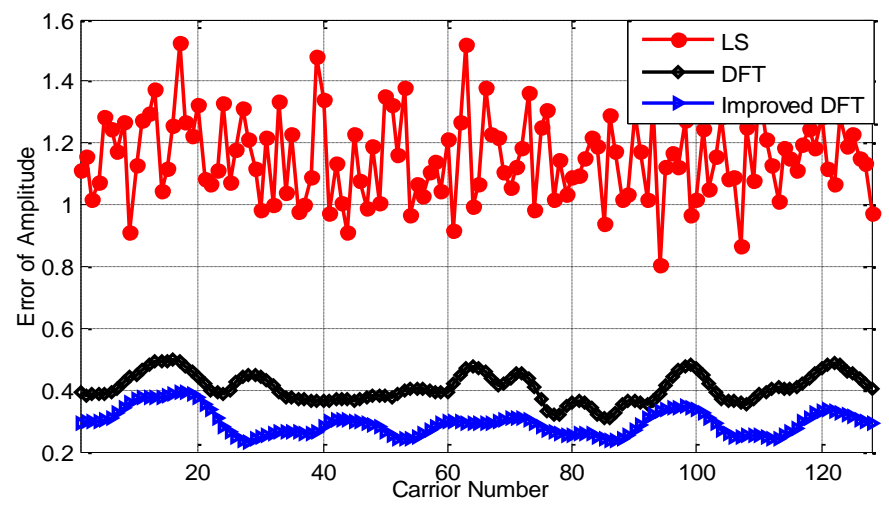

Fig. 7 Magnitude error of different estimation methods

\section{Concluthsion}

In this paper, we have proposed an improved LS channel estimation algorithm based on DFT time domain filtering to estimate channel in tunnel environment. The improved algorithm can reduce noises inside protection interval by using a dynamically changed parameter and get better performance in low signal-to-noise ratio. Because DFT can be realized through IFFT/FFT technology, so the improved algorithm also preserves the easy implementation and low complexity characteristics of the LS algorithm. In conclusion, the improved algorithm has good practical value and is suitable for tunnel environment channel estimation in low SNR conditions.

\section{References}

[1] Lin, Jia-Chin C. "LS Channel Estimation for Mobile OFDM Communications on Time-Varying Frequency Selective Fading Channels," IEEE Internation Conference, June 2007, pp. 3016-3023.

[2] J.-J. Van de Beek, O. Edfors, M. sandell, S. K. Wilson, and P. O. Borjesson, "on channel estimation in OFDM systems," in Proc. IEEE VTC'95, July 1995, vol. 2, pp. 815-819, 25-28.

[3] S. Coleri, M. Ergen A. Puri and A. Bahai, "Channel estimation techniques based on pilot arrangement in OFDM systems," IEEE Trans. Broadcast, Sep 2002, vol. 48, pp. 223-229.

[4] Zhi Sun, Ian F. Akyildiz, "Channel Modeling and Analysis for Wireless Networks in Underground Mines and Road Tunnels," IEEE Trans. June 2010, vol. 58, No. 6, pp. 1758-1768.

[5] M. Moreli, U. Mengali, "A comparison of pilot-aided channel estimation method for OFDM systems," IEEE Trans. On signal Processing, Dec 2001, vol. 49, No. 12, pp. 3065-3073.

[6] Haene, Simon, Burg, "FFT processor for OFDM Channel Estimation," IEEE ISCAS, May 2007, pp. 1417-1420.

[7] O. Edfors, M. sandell, J.-J. Van de Beek, S. K. Wilson, and P. O. Borjesson, "Analysis of DFT-based channel estimators for OFDM," Wireless Personal Commun. Jan 2000, vol. 12, pp. 55-70

[8] ZHENG Kan, ZENG Guo yan, WANG wen bo, "DFT-based uplink channel estimation in MC-CDMA systems," IEEE Eigth International Symposium on Spread Spectrum Techniques and Applications, Sep 2004, pp. 570-574. 